\title{
Potential combination endothelial progenitor cell and antiviral therapies for reversal of impaired angiogenesis induced by SARS-CoV-2 infection
}

Prasad S Koka ${ }^{1 *}$, Bharathi Ramdass ${ }^{1}$ and Srinivasa T Reddy ${ }^{2}$

*Correspondence: kokaprasad005@gmail.com

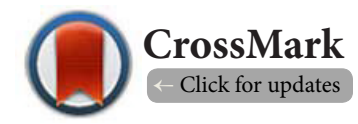

'Department of Virology \& Immunology, Haffkine Institute for Training, Research \& Testing, Acharya Donde Marg, Parel, Mumbai, Maharashtra 400012, India.

${ }^{2}$ Departments of Medicine and Molecular and Medical Pharmacology, David Geffen School of Medicine at UCLA, Los Angeles, California 90095, USA.

\begin{abstract}
Late stage detection of COVID-19 disease pathogenesis induced by SARS-CoV-2 infection may prove to be fatal to the infected patients with the severity increasing, if inhibition of differentiation of hematopoietic endothelial progenitor cells, or their sustained deficiency, is not reversed. Antiviral drug treatments of such individuals need not necessarily resuscitate from a severe impairment of normal angiogenesis of the vascular endothelium. The virus targets the endothelial progenitor cells which co-express the hematopoietic stem cell marker CD34 and the angiotensin converting enzyme 2 (ACE2), latter being the host receptor for this pathogen. There is not an apparent segregation of CD34 and ACE2 antigens, strongly implicating inhibition of differentiation of virus infected progenitor cells. The embattled clinical condition of SARSCoV-2 infected patients may well require a dual-mode endothelial progenitor cellular infusion and antiviral drug molecular therapies to stage a rapid clinical recovery. Umbilical cord blood derived CD34+ progenitor cells are the most optimal for rapid availability, harvesting and infusion into the severely ill infected patients, who are most likely to be non-responsive to solely antiviral drug treatments. This is about rapidly prepared allogeneic cell infusion therapy and not autologous cell transplantation which is impractical for these relatively acute and short-term conditions and treatments of SARS-CoV-2 infections. A combination progenitor cell and antiviral drug treatment is suggested for recovery and maintenance of normal angiogenesis of infected patients who may otherwise not survive. Hence the concept and its basis discussed needs to be urgently advanced to translation stage.
\end{abstract}

Keywords: SARS-CoV-2, CD34, CD133, endothelial progenitor cells, angiogenesis inhibition, transient treatment, progenitor cell therapy, neovascularization, thrombocytopenia, microRNA, iPSC, organoids

\section{Hypotheses}

Variations caused by SARS-CoV- 2 in levels of disease pathogenesis in infected patients from asymptomatic or minor symptoms, to severe damage to vascular cells, raises questions on the return to normal functioning of the vasculature and recuperation of the severely affected physiology of angiogenesis pathways.

The level or stage of the COVID-19 disease at which the patients' infection by SARS-CoV- 2 is tested to be positive by PCR for existing infection, is critical to determine the therapeutic strategy to be adopted. If SARS-CoV- 2 infection has advanced the virus induced disease to injury of the vasculature and its endothelium, eliciting an abnormally potent cytokine storm due to the severe but acute surge of immune responses, then recovery may need the dual administration of exogenous cellular in addition to the molecular drug therapies.

Umbilical cord blood (UCB) derived endothelial progenitor cells (EPCS) despite their allogeneic nature are evidenced as the most resourceful and generally safest for infusion into the patients [1], in this instance battling the severity of SARS-CoV-2 infection. However, it is important to distinguish between our 
suggested intravenous (IV) cell infusion for recovery and homeostasis of angiogenesis than undertake the impractical cell transplantation, in such a critical clinical scenario. Infusion can be performed following rapid cell preparation including any pre-IV HLA matching on a few UCB derived cells [1].

Herein a combination cellular and molecular therapy that has all the prerequisites for rapid clinical administration to the virus infected patients struggling between life and death is proposed. Figure 1 depicts the desirable therapeutic approach if the infected patient is already at the disease stage where she needs ventilator support to remain stable and for recovery.

Lung microvascular endothelial progenitor cells (EPCS) play a pivotal role in maintaining the life of an individual [2]. In our Figure 1, UCB derived endothelial progenitor cells expressing the characteristic CD34 antigen of the hematopoietic lineage (HEPC) $[1,3]$, may come to the rescue of replenishing the severely depleted endothelial cells of the vasculature of the critically ill and even non-responsive patients, arising from SARS-CoV-2 infection. However, such human umbilical cord hematopoietic endothelial progenitor cell (hUCHEPC) infusion still would need an anticipated antiviral drug treatment for containment of the virus replication, to aid in the HEPC/EPC homeostasis and stabilization for reversal of the otherwise aggravated patients' physiological condition.

Inhibitors of this viral RNA synthesis (from its +ssRNA strand), such as remdesivir and ED-1931/ED-2801, are very much in clinical trials with much anticipated efficacy thru' aborted viral replication, due to the incorporated modified nucleotides $[4,5]$. Viral protease inhibitors of SARS-CoV-2 serine protease are also being developed [6-9]. Therefore, for the infected individuals who become severely ill from damaged vasculature, in order to re-attain stable vascularization (neovascularization), hUCHEPC may be the most plausible therapy to be adopted [10-12], aided by the antiviral drugs [4-9], to achieve and sustain the normal angiogenesis recovery.

Binding of the SARS virus spike (S) protein to the endothelial cell angiotensin converting enzyme-2 (ACE2) [13], as it also happens with strong affinity to SARS-CoV-2 in particular, is physiologically deleterious to the host HEPC/EPC self-renewal and differentiation into mature cells of different types of the human vasculature [14-16]. The steady state disruption between the HEPC/EPC in effect during the virus infection also necessitates the antiviral drug induced containment and clinically eventual cessation of virus replication to maintain functioning levels of endothelial progenitor cells. Else, resurgence and function of the HEPC/EPC prevail during decreasing viral loads not requiring the cellular therapy component, presumably in the virus infected asymptomatic individuals, or patients that are responsive to antiviral drugs without clinical advancement to pneumonia and severe lung-vascular cell injury.

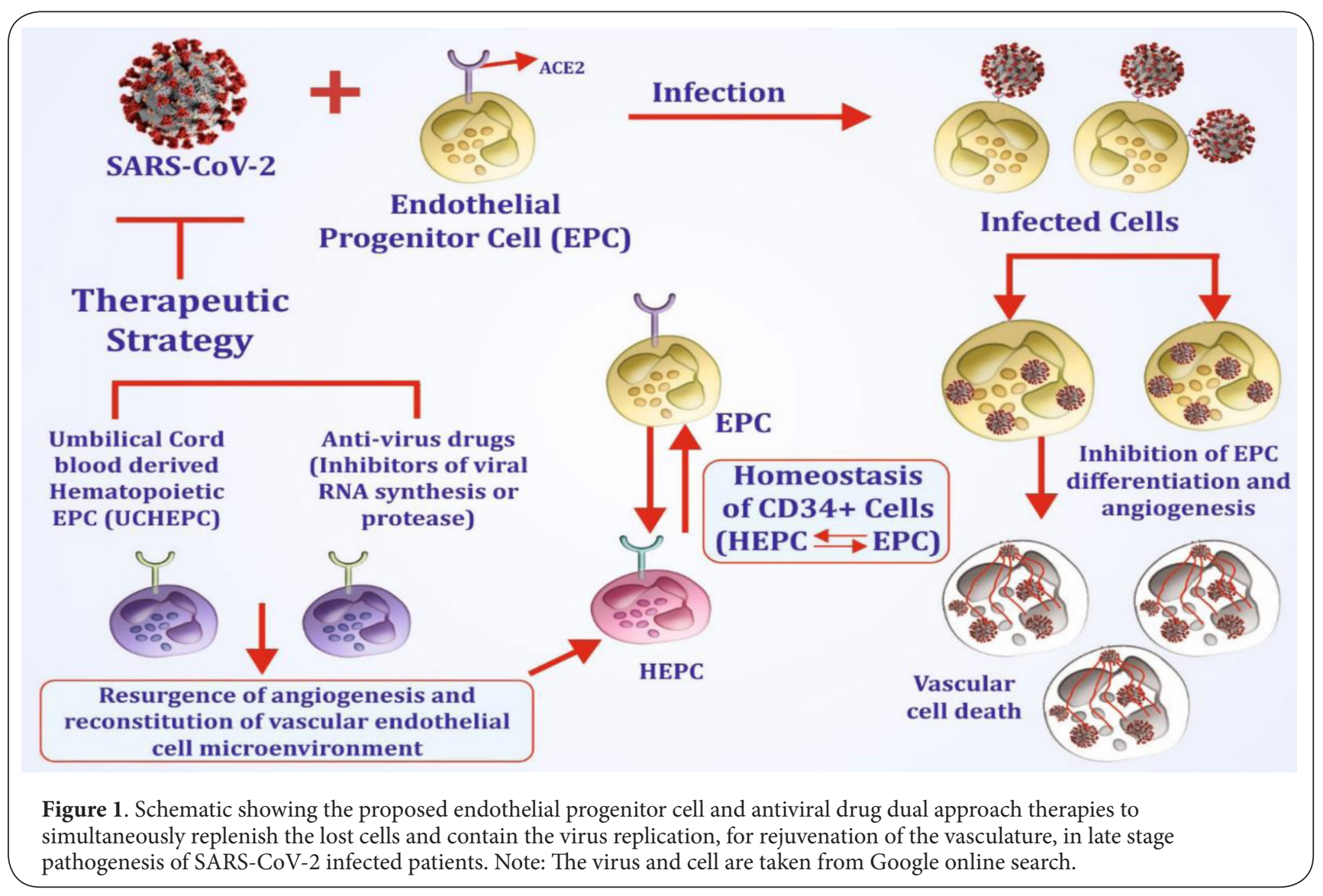


Despite the common haematopoietic lineage association of the endothelial progenitor cells that also express the characteristic hematopoietic cell surface CD34 antigen marker $[1,17]$, onset of stage specific divergent lineage commitment of HEPC and EPC (CD34+) lead them to differentiate separately into immune and vascular cells respectively. HEPC are in greater proximity to being stromal than EPC. Previously, we have performed engraftments and reconstitution of CD34+ progenitor cells, isolated rapidly by AutoMacs, in human stromal microenvironment in SCID-hu animals $[18,19]$. In the context of EPC, CD34 expression lends credence to the stromal proximity and coexistence between HEPC and EPC populations which are discriminated only through their lineage differentiation into cell types categorized by their functions designated prior to HEPC/EPC "split". Lung vascular EPC microenvironment [2] preservation is essential for the circulation of vascular cells which are the primary target of this virus and interruption or failure of this function is potentially lethal to the infected patients. UCB derived CD34+ H/EPCs [19] for the cellular therapy can be used for regeneration of the severely depleted vascular progenitor cells in the SARS-CoV-2 infected patients.

Moreover, purified CD34+ cells, even from peripheral blood are significantly more preventative of graft-versus-host disease (GVHD) in allogeneic transplantations [20]. Further, UCB derived CD34+ cells carry the potential to confer greater survival incidence and ward off GVHD related mortality in transplantation recipients [21]. Thus, when GVHD due to CD34+ cell transplantation is at a minimal in causing patient mortality, a transient readily preparative and administrable CD34+ cell infusion therapy that we have proposed is not expected to confer a burden on the immune system of the COVID-19 patients. Rather any such presumably mild reaction $[20,21]$ could be even synergistically beneficial in repressing or lowering any prevailing clinical cytokine storm condition in these SARSCoV-2 infected patients, in conjunction with some of the other prescribed and adopted treatments.

Interestingly, regions of the S/pike protein of SARS-CoV-2 cause cytokine storm induced thrombocytopenia, is similar to that effected by HIV-1 V3 loop determinants $[22,23]$. The commonality of virus-induced depletion of the megakaryocyte lineage CD41+ cells [24], may well strengthen the involvement of microRNA in the inhibition of differentiation of progenitor cells [25], H/EPC, causing insufficient supply of cells required to be maintained in normal blood circulation and in the case of both these viruses, for platelet formation and levels.

Our recent report wherein differential regulation between microRNAs, miR-15a and miR-24, is implicated in HIV-1 induced inhibition of differentiation of $\mathrm{CD} 34+$ hematopoietic progenitor cells occurs, purportedly via HOX, and thus ensue different lineage cytopenias [25]. Similarly, miRNA implicated phenomena are involved in EPC differentiation and these cells' regulatory signals on miR-141-3p (via Hesr1) and miR-126 (via VEGFR2) in destabilization of proteins controlling prevention of lung injury [26], and type 2 diabetes [27], respectively. Such findings compel us to anticipate microRNAs to become the focus of consideration for drug therapies to address depletion of progenitor cells. Further, microRNAs may lead to cell-free therapies possibly encapsulated in bio-scaffolds. Targeting or using microRNAs may further delineate strategies to tackle the virus pathogen or its deleterious clinical conditions.

Sustained maintenance of stromal microenvironments in the human vasculature [28] post-therapy, as depicted in Figure 1, pertaining to the homeostasis between HEPC and EPC balance of the cell surface antigens, such as the inevitable CD34 [26] marker expression in this regard, is of utmost underlying physiological necessity. The homeostasis between HEPC and EPC as we depicted in Figure 1, seems to be driven by the co-expression of CD133 and CD34 on the EPC, with retention of the hematopoietic origins, for a sustained replenishment of the double-positive (CD34+CD133+) EPC that participates in the angiogenesis or neovascularization [1]. Whether a selection enriched for the multi-potent ALDH+CD133+Linphenotype cells' repopulating potential as in in vivo [29], of these CD34+ subset cells when derived from the UCB, would be more efficacious for the H/EPC+ therapy for severe acute SARS-CoV-2 infections, needs to be determined. The vascular endothelium may have been depleted of the repopulation supporting stromal microenvironment in the SARS-CoV- 2 infected patients but then again, this ALDH+CD133+Lin-/CD34+ cell population enrichment [29] may very well be the cofactor in the midst of the whole CD34+ cells together providing the cellular microenvironment needed for rapid and irreversible neovascularization. These co-populating progenitor stem cell subset phenotypic requirements but led by the focus and ease to collect large numbers of the CD34+ cells from different UCB samples, will be expected to counter and recover the SARSCoV-2 induced damage to the vasculature. This will address the well-being of the affected patients in general, and SARSCoV-2 infected patients in particular, for attainment of the elimination of the entire and last vestiges of infection and the virus induced pathogenesis.

Thus we hope that basic science and clinical researchers may follow up on these suggested lines of therapeutic treatment to evaluate the feasibility and efficacy in severely ill COVID-19 patients to stage a recovery, not without a sense of urgency.

Furthermore, since SARS-CoV-2 infections also cause dysfunction of disparate human organs including the lungs, heart, brain, kidney, intestine, etc., disease modeling by employing the human induced pluripotent stem cells (hiPSC) derived organoidmodel systems [30-40], lead the path towards the mechanisms underlying organ failures due to SARS-CoV-2 infections. Such experimental investigations using hiPSC derived organoid models could well generate molecular drug therapies to treat preemptive tissue damage by SARS-CoV- 2 infections.

\section{Ethical considerations}

As this article deals only with presentation of perspectives on 
Koka et al. Stem Cell Biology and Research 2020,

http://www.hoajonline.com/journals/pdf/2054-717X-7-1.pdf

doi: 10.7243/2054-717X-7-1

the subject without any experimental, scientific and clinical investigations, including non-use or non-involvement of biological materials, or there were no recruitment of human subjects, therefore informed consent, ethics committee, or institutional review board (IRB) approvals and clinical trials registration are all not required.

\section{Competing interests}

The authors declare that they have no competing interests.

\section{Authors' contributions}

\begin{tabular}{|l|c|c|c|}
\hline Authors' contributions & PSK & BR & STR \\
\hline Research concept and design & $\checkmark$ & $\checkmark$ & -- \\
\hline Collection and/or assembly of data & $\checkmark$ & $\checkmark$ & -- \\
\hline Data analysis and interpretation & $\checkmark$ & -- & -- \\
\hline Writing the article & $\checkmark$ & -- & -- \\
\hline Critical revision of the article & $\checkmark$ & -- & $\checkmark$ \\
\hline Final approval of article & $\checkmark$ & -- & $\checkmark$ \\
\hline Statistical analysis & -- & -- & -- \\
\hline
\end{tabular}

\section{Acknowledgement}

This study was supported in part by a grant from NIA/NIH

R01 AG059502 (STR).

\section{Publication history}

Editor: Andreas K Nussler, University of Tubingen, Germany. Received: 17-July-2020 Final Revised: 12-Sept-2020

Accepted: 27-Sept-2020 Published: 05-Oct-2020

\section{References}

1. Janic B and Arbab AS. Cord blood endothelial progenitor cells as therapeutic andimaging probes. Imaging Med. 2012;4(4):477-490. doi:10.2217/iim.12.35.

2. Alvarez DF, Huang L, King JA, ElZarrad MK, Yoder MC, Stevens T. Lung microvascular endothelium is enriched with progenitor cells that exhibit vasculogenic capacity. Am J Physiol Lung Cell MolPhysiol2008;294:L419L430. doi:10.1152/ajplung.00314.2007.

3. Murohara T, Ikeda H, Duan J et al. Transplanted cord blood-derived endothelial precursor cells augment postnatal neovascularization. J Clin Invest. 2000;105(11):1527-1536.

4. Gordon CJ, Tchesnokov EP, WoolnerE. Remdesivir is a direct-acting antiviral that inhibits RNA-dependent RNA polymerase from severe acute respiratory syndrome coronavirus 2 with high potency. doi: $10.1074 / j b c$. RA120.013679jbc.RA120.013679.

5. Sheahan TP, Sims AC, Zhou S, et al. An orally bioavailable broad-spectrum antiviral inhibits SARS-CoV-2 in human airway epithelial cell cultures and multiple coronaviruses in mice.Sci. Transl. Med. 10.1126/scitranslmed. abb5883 (2020).

6. Dai W, Zhang B, Su H, et al. Structure-based design of antiviral drug candidates targeting the SARS-CoV-2 main protease. Science 22 Apr 2020

7. Tang $\mathrm{T}$, Bidon M, Jaimes JA, Whittaker GR, Daniel S. Coronavirus membrane fusion mechanism offers a potential target for antiviral development. Antiviral Research. 2020;178:104792.

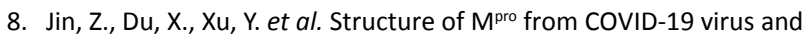
discovery of its inhibitors. Nature (2020). https://doi.org/10.1038/ s41586-020-2223-y

9. Wrapp D, Wang N, Corbett KS, et al. Cryo-EM structure of the 2019-nCoV spike in the prefusion conformation. Science 2020:367(6483):1260-1263. DOI: 10.1126/science.abb2507

10. Asahara T, Murohara T, Sullivan A, et al. Isolation of Putative Progenitor Endothelial Cells for Angiogenesis. Science. 1997;275(5302):964967. doi:10.1126/science.275.5302.964.
11. Kalka C, Masuda $\mathrm{H}$, Takahashi T, et al. Transplantation of ex vivo expanded endothelial progenitor cells for therapeutic neovascularization. PNAS 2000;97(7):3422-3427.

12. Sukmawati D, Tanaka R. Introduction to next generation of endothelial progenitor cell therapy: a promise in vascular medicine. Am J Transl Res 2015;7(3):411-421.

13. Hammingl, Timens W, Bulthuis MLC, Lely AT, Navis GJ, van Goor H. Tissue Distribution of ACE2 Protein, the Functional Receptor for SARS Coronavirus. A First Step in Understanding SARS Pathogenesis. J Pathol. 2004;203(2):631-637. doi: 10.1002/path.1570.

14. Chong MSK, Ng WK, Chan JKY. Concise Review: Endothelial Progenitor Cells in Regenerative Medicine: Applications and Challenges, Stem Cells Trans Med. 2016;5:530-538

15. Park S, Tepper OM, Galiano, RD. Selective Recruitment of Endothelial Progenitor Cells to Ischemic Tissues with Increased Neovascularization. Plastic and Reconstructive Surgery. 2004;113(1):284-293. doi: 10.1097/01.PRS.0000091169. 51035.A5

16. Ribatti D. The discovery of endothelial progenitor cells: An historical review. Leukemia Res. 2007;31(4):439-444. https://doi.org/10.1016/j. leukres.2006.10.014.

17. Zulli A, Rai S, Buxton BF, Burrell LM, Hare DL. Co-localization of angiotensin-converting enzyme2-, octomer-4- and CD34-positive cells in rabbit atherosclerotic plaques. ExpPhysio/93.5 pp 564-569

18. Koka PS, Kitchen CM, Reddy ST. Targeting c-Mpl for revival of human immunodeficiency virus type 1-induced hematopoietic inhibition when CD34+ progenitor cells are re-engrafted into a fresh stromal microenvironment in vivo. J Virol 2004;78:11385-11392.

19. Zhang M, Poh TY, Louache F, Sundell IB, Yuan J, Evans S, Koka PS. Rescue of multi-lineage hematopoiesis during HIV-1 Infection by human c- $\mathrm{mpl}$ gene transfer and reconstitution of CD34+ progenitor cells in vivo. J Stem Cells 2009; 4(3): 161-177.

20. Lang $P$, Handgretinger $R$, Niethammer D, Schlegel PG, Schumm M, Greil J, Bader P, Engel C, Scheel-Walter H, Eyrich M, Klingebiel T. Transplantation of highly purified CD34+ progenitor cells from unrelated donors in pediatric leukemia. Blood. 2003;101(4):1630-6.doi: 10.1182/ blood-2002-04-1203. Epub 2002 Oct 10.

21. MacMillan ML,Weisdorf DJ, Brunstein CG, Cao Q, DeFor TE, Verneris MR, Blazar BR, Wagner JE. Acute graft-versus-host disease after unrelated donor umbilical cord blood transplantation: analysis of risk factors. Blood. 2009; 113(11): 2410-2415.Clinical Trials and Observations. doi: 10.1182/blood-2008-07-163238.

22. Mehta P, McAuley DF, Brown M, Sanchez E, Tattersall RS, MansonJJ. COVID-19: consider cytokine storm syndromes and immunosuppression. 2020:1033-1034. https://doi.org/10.1016/S0140-6736(20)30630-9.

23. Zhang M, Evans S, Yuan J, Ratner L, Koka PS. HIV-1 determinants of thrombocytopenia at the stage of $\mathrm{CD} 34+$ progenitor cell differentiation in vivo lie in the viral envelope gp120 V3 loop region. Virology 2010;401(2):131-136.

24. Sundell IB, Koka PS. Thrombocytopenia in HIV infection: impairment of platelet formation and loss correlates with increased c-Mpl and ligand thrombopoietin expression. Curr HIV Res 2006;4(1):107-116.

25. Padmanabhan U, Dahake R, Chowdhary A, Koka PS. HIV-1 inhibits haematopoiesis via microRNA secreted by virus-infected CD4+ T-cells.Eur J Haematol 2019 doi:10.1111/ejh.13350

26. Jin Y, Liu W, Liu X, Ma T, Yang C, Cai Q, Liu Z. Transplantation of endothelial progenitor cells attenuated paraquat-induced acute lung injury via miR-141-3p-Notch-Nrf2 axis. Cell \& Bioscience 2018; 8: Article No.21.

27. Wu K, Yang Y, Zhong Y, et al. The effects of microvesicles on endothelial progenitor cells are compromised in type 2 diabetic patients via downregulation of the miR-126/VEGFR2 pathway. Am J PhysiolEndocrinol Metab2016;310:E828-E83. doi:10.1152/ajpendo.00056.2016.

28. Suzuki T, Sasano H, Takaya R, Fukaya T, Yajima A, Nagura H. Cyclic changes of vasculature and vascular phenotypes in normal human ovaries. Human Reproduction 1998;13(4):953-959.

29. Hess DA, Wirthlin L, Craft TP, Herrbrich PE, Hohm SA, Lahey R, Eades 
Koka et al. Stem Cell Biology and Research 2020,

WC, Creer MH, Nolta JA. Selection based on CD133 and high aldehyde dehydrogenase activity isolates long-term reconstituting human hematopoietic stem cells. Blood. 2006; 107(5): 2162-2169. doi: 10.1182/ blood-2005-06-2284.

30. Sachs N, Papaspyropoulos A, Zomer-van Ommen DD , Heo I, Böttinger $L$, et al. Long-term expanding human airway organoids for disease modeling. EMBO Journal 2019; 38: e100300.DOI 10.15252/ embj.2018100300.

31. Porotto M, Ferren M, Chen Y-W, Siu Y, Makhsous N, Rima B, Briese T, Greninger AL, Snoeck H-W, Moscona A. Authentic modeling of human respiratory virus infection in human pluripotent stem cell-derived lung organoids. mBio. 2019; 10:e00723-19. https://doi.org/ 10.1128/ mBio.00723-19.

32. Chen Y-W, Huang SX, de Carvalho ALRT, S-H, Islam MN, Volpi S, et al. A three-dimensional model of human lung development and disease from pluripotent stem cells.Nat Cell Biol. 2017; 19(5): 542-549. doi:10.1038/ ncb3510.

33. Israeli Y, Gabalski M, Ball K, Wasserman A, Zou J, Ni G, Zhou C, Aguirre A.Generation of heart organoids modeling early human cardiac development under defined conditions. bioRxiv 2020 doi: https://doi. org/10.1101/2020.06.25.171611.

34. Nugraha B, Buono MF, von Boehmer L, Hoerstrup SP. Emmert MY. Human Cardiac Organoids for Disease Modeling. ClinPharmacolTher. 2019;105(1):79-85.doi: 10.1002/cpt.1286. Epub 2018 Dec 21.

35. Kauffman AL, Ekert JE, Gyurdieva AV, Rycyzyn MA, Hornby PJ. Directed differentiation protocols for successful human intestinal organoids derived from multiple induced pluripotent stem cell lines. Stem Cell Biology and Research 2015. doi: 10.7243/2054-717X-2-1.

36. Bonventre JV. Kidney organoids-a new tool for kidney therapeutic development.Kidney Int. 2018; 94(6):1040-1042.doi: 10.1016/j. kint.2018.07.029.

37. Morizane R, Bonventre JV.Kidney Organoids: A Translational Journey Trends.Mol Med. 2017;23(3):246-263.doi: 10.1016/j. molmed.2017.01.001. Epub 2017 Feb 7.

38. Nishinakamura R. Human kidney organoids: progress and remaining challenges. Nature Reviews Nephrology 2019; 15, 613-624.

39. SteichenC ' Giraud S, Hauet T.Kidney organoids. Med Sci (Paris) 2019;35(5):470-477.doi: 10.1051/medsci/2019090. Epub 2019 May 22.

40. Takasato M, Er PX, Chiu HS, Maier B, Baillie GJ, Ferguson C, Parton RG, Wolvetang EJ, Roost MS, Chuva de Sousa Lopes SM, MH. Kidney organoids from human iPS cells contain multiple lineages and model human nephrogenesis. Nature 2015; 526, 564-568.

\section{Citation:}

Koka PS, Ramdass B and Reddy ST. Potential combination endothelial progenitor cell and antiviral therapies for reversal of impaired angiogenesis induced by SARS-CoV-2 infection. Stem Cell Biol Res. 2020; 7:1. http://dx.doi.org/10.7243/2054-717X-7-1 